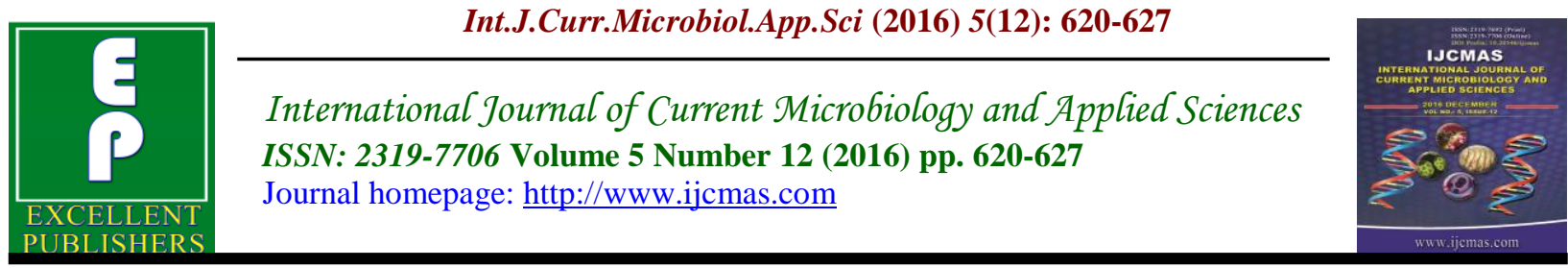

Original Research Article

http://dx.doi.org/10.20546/ijcmas.2016.512.068

\title{
Evaluation of different Staining Techniques in the Diagnosis of Trichomonas vaginalis Infection in Females of Reproductive Age Group
}

\author{
T. Shyamalajanaki, G. Sucilathangam*, G. Velvizhi and C. Revathy \\ Department of Microbiology, Tirunelveli Medical College, Tirunelveli - 627 011, \\ Tamil Nadu, India \\ *Corresponding author
}

\begin{abstract}
A B S T R A C T
Keywords

Trichomonas vaginalis,

Vaginal discharge,

Giemsa staining, Acridine orange staining.

\section{Article Info}

Accepted:

20 November 2016 Available Online:

10 December 2016

This prospective study was conducted in Department of Microbiology and Outpatient Department of Obstetrics and Gynaecology at Tirunelveli Medical College Hospital, Tirunelveli, and Tamil Nadu from June' 2015 and July' 2015.Out of 95 female patients of reproductive age group (15-45 years),Trichomonas vaginalis infection was detected in 16 patients (16.8\%).Vaginal discharge was the most prevalent symptoms, found in twenty two patients (23.2\%).The highest positivity was found in the age group 25-35 years which represent the group of high sexual activity. Wet mount examination for T. vaginalis was positive in $6.3 \%$ of cases, whereas, Giemsa staining and Acridine orange staining were positive in $10.5 \%$ and $16.8 \%$ of cases respectively. Although wet mount examination is the most commonly used test in routine diagnosis of Trichomonas vaginalis infection, but staining techniques should be used as an additional diagnostic test in order to diagnose cases missed either due to unavailability of immediate microscopic facility or delay in the transport of samples to the laboratory for culture.
\end{abstract}

\section{Introduction}

Trichomonas vaginalis, a parasitic protozoan, is the etiologic agent of Trichomoniasis, a sexually transmitted disease (STD) of worldwide importance. Trichomoniasis is the most common non viral STD, and it is associated with many perinatal complications, male and female genitourinary tract infections, and an increased incidence of HIV transmission (Brown, 1972; Catterall, 1972). Trichomoniasis is one of the most common sexually transmitted infections (STIs) with a prevalence of $5-75 \%$. In India, Trichomoniasis accounts for $2-7 \%$ of all
STIs. Infection with Trichomonas vaginalis is known to cause vaginitis (Sood et al., 2007; Kumarasamy et al., 2008).

Given the public health implications of $T$. vaginalis infection in the mainstream reproductive age population, there is a need rethink current public health policy on this easily treatable STI. Existing strategies focus on high-risk populations, ignoring the bulk of the disease burden in India. Furthermore, treatment guidelines use syndromic management of RTI/STI, an ineffective approach when almost half of $T$. 
vaginalis infections are asymptomatic and there is no provision for partner treatment.

Diagnosis is difficult, since the symptoms of Trichomoniasis mimic those of other STDs and detection methods lack precision. With increasing availability of simple and inexpensive point-of-care tests for $T$. vaginalis infection, there is a growing need for further evaluation and implementation of point-of-care screening particularly in settings where young women seek healthcare. Because this infection increases the risk of HIV transmission and is associated with adverse pregnancy outcomes, there is a need for increased screening and treatment of this easily curable sexually transmissible infection in India. There is a dearth of data on the prevalence and risk factors for $T$. vaginalis infection among women in India.

Hence, the present study was undertaken to determine the prevalence of Trichomonas vaginalis Infection and also to evaluate the efficacy of staining procedures like Giemsa staining and Acridine orange staining in comparison with wet mount examination for the diagnosis of Trichomonas vaginalis infection in females of reproductive age group.

\section{Materials and Methods}

This prospective study was conducted Department of Microbiology and Outpatient Department of Obstetrics and Gynaecology at Tirunelveli Medical College Hospital, Tirunelveli, and Tamil Nadu during the period of June' 2015 and July' 2015.The study protocol was approved by the Institutional Scientific and Ethics Committee.

\section{Study population}

Female patients of reproductive age group (15-45 years) with complaints of foul smelling vaginal discharge, pruritis, dyspareunia, dysuria, and pain in lower abdomen.

\section{Sample Collection and Processing}

Samples were collected from 100 women of childbearing age in the Outpatient Department of Obstetrics and Gynaecology. Sociodemographic variables included age, education, religion, marital status, monthly household income, occupation, and availability of a toilet at home were collected. Vaginal discharge was taken from the posterior cervix or from the vaginal wall using a sterile swab containing Stuart's transport media. An oral informed consent was taken from each patient and two vaginal swabs were taken from posterior fornix. One was used to prepare wet mount and second was used to prepare smear for Giemsa staining and Acridine orange staining.

\section{Laboratory procedures}

\section{Wet mount examination}

Wet mount was prepared using $0.85 \%$ physiological saline and was examined with a light microscope at $10 \mathrm{X}$ and $40 \mathrm{X}$. The Trichomonads were identified by their size $(10-20 \mu \mathrm{m})$, round or oval shape, and characteristic quivering or twitching motility (Mason et al., 2001).

\section{Giemsa Staining}

The prepared smear was fixed by immersion in methanol for one minute and allowed to dry. It was then stained with Giemsa stain (Hi Media Laboratories, India) and was scanned for Trichomonas vaginalis at $100 \mathrm{X}$ magnification.

Both the internal and external structures of the organism were clearly visualized. The former stained dark blue with a red nucleus 
and the latter was sharply outlined, showing clearly the flagella and the undulating membrane (van Der Schee et al., 1999).

\section{Acridine Orange Staining}

The prepared smear was fixed with methanol for 2-3 min and covered with freshly prepared acridine orange dye (Hi Media Laboratories, India) in a concentration of $5 \mathrm{mg} / \mathrm{ml}$ which was then left at room temperature for $2 \mathrm{~min}$. After being rinsed with distilled water, the slide was examined under a fluorescence microscope (with a 470-490 nm filter) at a magnification of 40X.Epithelial cells were fluoresced light green with a bright green nucleus. The nuclei of leukocytes (pus cells) were fluoresced bright green and bacteria were stained bright red. The trophozoites of Trichomonas vaginalis were seen as characteristic brick red color with a yellowish green nucleus.

\section{Results and Discussion}

A total of 95 female patients of reproductive age group (15-45 years) were included in the study. Ninety five Symptomatic patients were included of which; 20 were infertile \& 75 patients were multiparous women. The mean age of symptomatic patients was 32.6 years (range 15-45).

Vaginal discharge was the most prevalent symptoms, found in twenty two patients $(23.2 \%)$, while 14 patients $(14.7 \%)$ had discharge and itching, 14 patients (14.7\%) had discharge and dysuria, 14 patients (14.7\%) had discharge, itching and dysuria, discharge, itching, 8 patients $(8.4 \%)$ had dysparenuia and dysuria and 23 (24.3\%) had Discharge and lower abdominal pain(Table1).Distribution of $T$. vaginalis infection according to age groups was depicted in.(Table-2)
Direct microscopic examination of smears from vaginal swab detected 16 positive cases of Trichomoniasis. Wet mount examination for $T$. vaginalis was positive in $6.3 \%$ of cases, whereas, Giemsa staining and Acridine orange staining were positive in $10.5 \%$ and $16.8 \%$ of cases respectively [Table-3].

Out of total 16 positive cases, 6 cases were positive in wet mount examination. The sensitivity of wet mount examination was found to be $37.5 \%$. A correlation was done between the different diagnostic tests performed and it was found that out of 16 positive cases, Giemsa staining detected 4 wet mount negative cases, whereas, Acridine orange staining detected 10 wet mount negative cases along with 6 Giemsa negative cases [Figure-1] .

The efficacy of staining techniques was determined in comparison with wet mount examination and sensitivity, specificity, positive predictive value (PPV) and negative predictive value (NPV) of Giemsa staining and Acridine orange staining were calculated [Table-4].

Trichomoniasis is an important public health disease that is associated with vaginitis, cervicitis, urethritis, pelvic inflammatory disease (PID) and in some cases infertility. It plays a role in the acquisition and transmission of the human immunodeficiency virus (HIV). In pregnant women trichomonads may be associated with the premature rupture of membranes, premature delivery, and delivery of lowbirth weight infants.

The prevalence of $T$. vaginalis is likely to be underestimated because there are no guidelines for $T$. vaginalis screening of women and clinicians often rely upon insensitive diagnostic methods. Screening 
for Trichomonas infection has been performed using direct microscopy or culture of the organism. However, many programs do not include Trichomonas infection screening.

The prevalence of Trichomonas is reported to be varying, in the present study, it was $16.8 \%$ which is similar to other studies ranging from $1.3 \%$ to $13.3 \%(8)$.The highest positivity was found in the age group 25-35 years which represent the group of high sexual activity. The present study has been noted that $T$. vaginalis infection occurs at ages of greatest sexual activity, this may agrees with many workers. $(9,10)$ The percentage of positive cases may correlate with sexual activity so the positive cases of $T$. vaginalis tends to decrease with advanced age as compared to young sexually active women.

Diagnosis of trichomoniasis is usually based on the presence of clinical symptoms such as vaginal discharge, irritation, burning sensation, dysuria and vaginal $\mathrm{pH}$. Vaginal discharge was the most prevalent symptoms, found in twenty two patients (23.2\%), in the present study. This is comparable to other studies El-Gayar and Rashwan, reported vaginal discharge and itching in their symptomatic female patients to be $(100 \%)$ and $(54.4 \%)$ respectively (El-Gayar et al., 2007).

Wet mount examination is the most frequently used method for diagnosis of Trichomoniasis in women. In this study, out of the 95 patients tested, $6(6.3 \%)$ cases were positive for $T$ vaginalis infection by wet mount examination with sensitivity of $37.5 \%$. The low sensitivity of wet mount diagnosis of Trichomonas in the present study $(37.5 \%)$ is very similar to other studies reported sensitivity of wet mount was; $58.5 \%, 61.9 \%$, respectively. (Lawing et al., 2000; Huppert et al., 2007). The reason for this is probably due to deterioration which leads the Trichomonads to lose motility, retract flagella, change morphology by becoming rounder and thereafter become difficult to distinguish from similar structures, such as leucocytes. In addition the examination should be done immediately after collection of specimen and needs experience and access to microscopy.

It has been reported that the sensitivity of wet mount examination ranges between 35$80 \%$ depending on the technical expertise of the observer. In one of the study it was shown that the sensitivity and specificity of direct wet mount microscopy was $95.83 \%$ and $100 \%$ respectively, in comparison to culture (Fernando et al., 2011). Although, positive wet mount is diagnostic, a negative test cannot exclude trichomoniasis because of low sensitivity (Swygard et al., 2004). It is reported that a minimal concentration of 104 organisms per milliliter of vaginal fluid is necessary for the identification of this protozoan by wet mount as low number of Trichomonas can be easily missed when they are in the presence of large number of leukocytes. Since the protozoa lose their distinctive motility on cooling to room temperature, a microscope and an experienced microscopist must be readily available in the clinical setting and the specimen should be examined as quickly as possible.

The performance of Giemsa staining was good and it was able to detect Trichomonas vaginalis in $10(10.5 \%)$ out of 95 patients, with a sensitivity of $66.6 \%$ and specificity of $40 \%$. Studies done by different workers have reported varying range of sensitivity (41-56\%) of Giemsa stained smears for the diagnosis of Trichomonas vaginalis infection (Radonjic et al., 2006).

In one study it was reported that Giemsa staining had high sensitivity (100\%) and 
specificity (99.69\%) compared to culture. The high sensitivity (100\%) reported in their study was attributed to centrifugation of sample prior to preparation of smears which led to concentration of large number of parasites. In set up lacking immediate microscopic facilities Giemsa staining is very useful, where prepared and fixed smears can be transported to the laboratory for diagnosis.

Table.1 The distribution of study population according to clinical symptoms

\begin{tabular}{|l|c|c|}
\hline Clinical Symptoms & $\begin{array}{c}\text { No of } \\
\text { patients }\end{array}$ & $(\mathbf{\% )}$ \\
\hline Discharge only & 22 & $23.2 \%$ \\
\hline Discharge and itching & 14 & $14.7 \%$ \\
\hline Discharge and dysuria & 14 & $14.7 \%$ \\
\hline Discharge, itching and dysuria & 14 & $14.7 \%$ \\
\hline Discharge, itching, dysparenuia and dysuria & 8 & $8.4 \%$ \\
\hline Discharge and lower abdominal pain & 23 & $24.3 \%$ \\
\hline Total & 95 & $100 \%$ \\
\hline
\end{tabular}

Table.2 Distribution of T. vaginalis infection according to age groups

\begin{tabular}{|c|c|c|c|c|c|}
\hline Age Group & Positive & $\mathbf{( \% )}$ & Negative & $\mathbf{( \% )}$ & Total \\
\hline $15-25$ & 3 & $3.1 \%$ & 11 & $11.6 \%$ & 14 \\
\hline $25-35$ & 11 & $11.6 \%$ & 51 & $53.7 \%$ & 62 \\
\hline $35-45$ & 2 & $2.1 \%$ & 17 & $17.9 \%$ & 19 \\
\hline Total & 16 & $16.8 \%$ & 79 & $83.2 \%$ & 95 \\
\hline
\end{tabular}

Table.3 Detection of Trichomonas vaginalis by wet mount examination and staining techniques

\begin{tabular}{|l|c|c|c|c|}
\hline \multicolumn{1}{|c|}{ Test done } & Positive cases & $\boldsymbol{\%}$ & Negative cases & $\boldsymbol{\%}$ \\
\hline Wet mount & 6 & $6.3 \%$ & 89 & $93.7 \%$ \\
\hline Giemsa stain & 10 & $10.5 \%$ & 85 & $89.5 \%$ \\
\hline Acridine orange & 16 & $16.8 \%$ & 79 & $83.2 \%$ \\
\hline
\end{tabular}

Table.4 Efficacy of Giemsa staining and Acridine orange staining in comparison with wet mount examination

\begin{tabular}{|l|c|c|c|c|}
\hline $\begin{array}{l}\text { Staining } \\
\text { techniques } \\
\text { performed }\end{array}$ & Sensitivity (\%) & Specificity (\%) & $\begin{array}{c}\text { Positive } \\
\text { predictive } \\
\text { value (\%) }\end{array}$ & $\begin{array}{c}\text { Negative } \\
\text { predictive } \\
\text { value (\%) }\end{array}$ \\
\hline Giemsa staining & $66.6 \%$ & $40 \%$ & $40 \%$ & $66.6 \%$ \\
\hline $\begin{array}{c}\text { Acridine Orange } \\
\text { staining }\end{array}$ & $100 \%$ & $71.4 \%$ & $50 \%$ & $100 \%$ \\
\hline
\end{tabular}


Fig.1

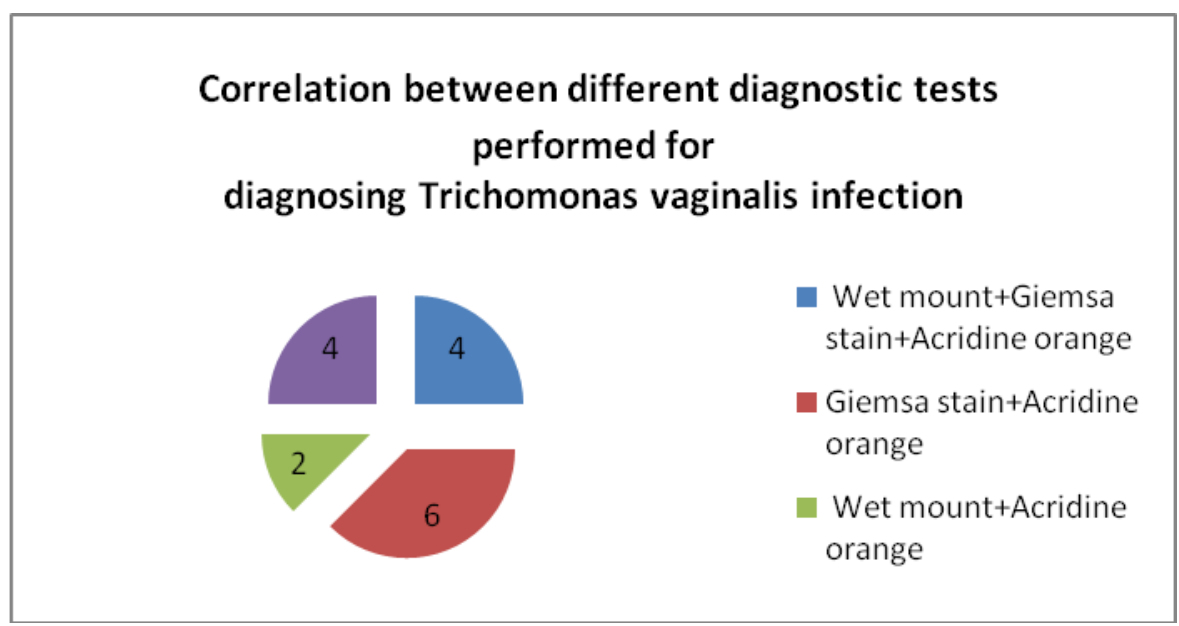

Also, with large number of patients attending gynecological outpatient department, an immediate examination of a vaginal swab is virtually impossible. Unlike, wet mount examination, delay in transport has no significant impact on its reliability for diagnosing Trichomonas vaginalis. However, it is time consuming and needs technical expertise.

Acridine orange staining was positive in 16 $(16.8 \%)$ out of 95 patients included in the study, with sensitivity and specificity of $100 \%$ and $71.4 \%$ respectively. Whereas, in one study acridine orange staining showed a high sensitivity and specificity of $71.43 \%$ and $99.44 \%$ respectively, in another study its sensitivity was only $47.5 \%$ in comparison to culture (Cevahir et al., 2002). Also, it has been reported that the sensitivity of acridine orange staining is high $(67 \%)$ in women with T.vaginalis infection alone, and low $(53 \%)$ in women with multiple infections (Bickley et al., 1989). In the present study, maximum number of cases of vaginal trichomoniasis was detected by acridine orange staining. This is in agreement with a previously done study which showed that the infection rate detected by acridine orange staining was higher than either culture or other vaginal swab examinations.
Although Acridine orange staining is easy to perform, rapid and screening of the vaginal smears is done in less time, the major disadvantage of this technique is that it requires special microscopic facilities, trained personnel and availability of the dye. Also, the smears lose their fluorescence with the passage of time, so permanent record is not possible. However, the rapidity, ease and reliability of acridine orange staining justify its use in routine laboratory diagnosis of Trichomonas infection.

In conclusion, acridine orange staining was found to be the best microscopic method when compared with wet mount examination and Giemsa staining. Hence, it should be used in routine diagnosis of Trichomonas vaginalis infection in places where fluorescent microscopic facility is available. This will also provide rapid screening of vaginal smears in patients suffering from vaginal discharge and thus, help in early diagnosis and prompt treatment of patients.

\section{References}

Agarwal, S., Sharma, V., Sarin, R. 2005. Reproductive tract infections in women- Prevalence, HIV 
seropositivity and role of conventional methods in diagnosis. Indian J. Sex Transm. Dis., 26(2): 73-77.

Ali, A.Q.M. 2014. Epidemiology Study of T. vaginalis in Babylon Province and efficiency of Mentha spicata leafs extracts in vivo. J. Nat. Sci. Res., 4(2): 72-82.

Al-Zabady, S.W.K. 2004. Isolation and Identification of Trichomonas vaginalis from trichomoniasis patients in Najaf city. M.Sc. Thesis, College of education, Kufa University, 75.

Beverly, A.L., Vengtarik, L.M., Cotton, B., Schwebke, J.R. 1999. Viability of Trichomonas vaginalis in transport medium. J. Clin. Microbiol., 37: 374950.

Bickley, L.S., Krisher, K.K., Punsalang, A. Jr, Trupei, M.A., Reichman, R.C., Menegus, M.A. 1989. Comparison of direct fluorescent antibody, acridine orange, wet mount, and culture for detection of Trichomonas vaginalis in women attending a public sexually transmitted diseases clinic. Sex Transm Dis., 16(3): 127-31.

Brown, M.T. 1972. Trichomoniasis. Practitioner, 209: 639-644.

Catterall, R.D. 1972. Trichomonal infections of the genital tract. Med. Clin. North Am., 56: 1203-1209.

Cevahir, N., Kaleli, I., Kaleli, B. 2002. Evaluation of direct microscopic examination, acridine orange staining and culture methods for studies of Trichomonas vaginalis in vaginal discharge specimens. Mikrobyol Bul., 36: 329-35.

Clark, D.H., Solomons, E. 1959. An evaluation of routine culture examination for Trichomonas vaginalis and Candida. Am. J. Obstet. Gynecol., 78: 1314-9.

El-Gayar, E.K. and Rashwan, M.F. 2007. Cervical intraepithelial neoplasia
(CIN) and Trichomonas vaginalis infection as revealed by polymerase chain reaction. J. Egypt Soc. Parasitol., 37(2): 623-630.

Fernando, S.D., Herath, S., Rodrigo, C,. Rajapakse, S. 2011. Improving diagnosis of Trichomonas vaginalis infection in resource limited health care settings in Sri Lanka. J. Glob. Infect. Dis., 3(4): 324-28.

Huppert, J.S., Mortesen, J.E., Reed, J.L, Kahn, J.A., Rich, K.D., Miller, W.C. et al. 2007. Rapid antigen testing compares favorably with transcriptionmediated amplification assay for detection of Trichomonas vaginalis in young women. Clin. Infec. Dis., 45(2): 194-198.

Krieger, J.N., Tam, M.R., Stevens, C.E., Nielsen, I.O., Hale, J., Kiviat, N.B., et al. 1988. Diagnosis of trichomoniasis: Comparison of conventional wetmount examination with cytologic studies, cultures, and monoclonal antibody staining of direct specimens. JAMA, 259(8): 1223-27.

Kumarasamy, N., Balakrishnan, P., Venkatesh, K.K., Srikrishnan, A.K., Cecelia, A.J., Thamburaj, E., et al. 2008. Prevalence and incidence of sexually transmitted infections among South Indians at increased risk of HIV infection. AIDS Patient Care STDS, 22: 677-82.

Kurth, V.A., Whittington, W.L., Golden, M.R., Thomas, K.K., Holmes, K.K. and Schwebk, J.R. 2004. Performance of a new, rapid assay for detection of Trichomonas vaginalis. J. Clin. Microbiol., 42(7): 2940-2943.

Lawing, L.F., Hedges, S.R. and Schwebke, J.R. 2000. Detection of trichomoniasis in vaginal and urine specimens from women by culture and PCR. J. Clin. Microbiol., 38(10): 3585-3588. 
Mason, P.R., Gregson, S., Gwanzura, L., Cappuccinelli, P., Rapelli, P., Fiori, P.L. 2001. Enzyme immunoassay for urogenital trichomoniasis as a marker of unsafe sexual behaviour, Epidemiol. Infect., 126: 103-109.

Mason, P.R., Super, H., Fripp, P.J. 1976. Comparison of four techniques for the routine diagnosis of Trichomonas vaginalis infection. J. Clin. Path., 29: 154-57.

Petrin, D., Delgaty, K., Bhatt, R., Garber, G. 1998. Clinical and microbiological aspects of Trichomonas vaginalis. Clin. Microbiol. Rev., 11(2): 300-17.

Radonjic, I.V., Dzamic, A.M., Mitrovic, S.M., Arsenijevic, V.S.A., Popadic, D.M., Zec, I.F.K. 2006. Diagnosis of Trichomonas vaginalis infection: The sensitivities and specificities of microscopy, culture and PCR assay. Eur. J. Obstet. Gynecol. Repro Biol., 126(1): 116-20.

Sood, S., Mohanty, S., Kapil, A., Tolosa, J., Mittal, S. 2007. In Pouch TV culture for detection of Trichomonas vaginalis. Indian J. Med. Res., 125: 567-71.

Sutton, M., Sternberg, M., Koumans, E.H., McQuillan, G., Berman, S., Markowitz. 2007. The Prevalence of Trichomonas vaginalis infection among reproductive-age women in the united states, 2001-2004. Clin. Infect. Dis., 45(10): 1319-26.

Swygard, H., Sena, A.C., Hobbs, M.M., Cohen, M.S. 2004. Trichomoniasis: clinical manifestations, diagnosis and management. Sex Transm. Infect., 80: 91-95.

van Der Schee, C., van Belkum, A., Zwijgers, L., van Der Brugge, E., O'neill, E.L., Luijendijk, A., et al. 1999. Improved Diagnosis of Trichomonas vaginalis Infection by PCR Using Vaginal Swabs and Urine Specimens Compared to Diagnosis by Wet Mount Microscopy, Culture, and Fluorescent Staining, J. Clin. Microbiol., 37(12): 4127-30.

\section{How to cite this article:}

Shyamalajanaki, T., G. Sucilathangam, G. Velvizhi and Revathy, C. 2016. Evaluation of different Staining Techniques in the Diagnosis of Trichomonas vaginalis Infection in Females of Reproductive Age Group. Int.J.Curr.Microbiol.App.Sci. 5(12): 620-627. doi: http://dx.doi.org/10.20546/ijcmas.2016.512.068 